ISSN: 2637-773X

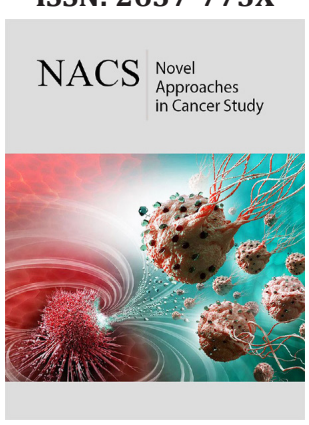

*Corresponding author: Daniel Borsch, Assistant Professor, LECOM at Seton Hill Greensburg, Pennsylvania, USA

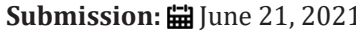

Published: 酸July 09, 2021

Volume 6 - Issue 2

How to cite this article: Kevin Pak, Johnny Dang, Michaela LeDonne, Daniel Borsch, et al. A Review of the Potential Preventive Effects of Adiponectin in Major Cancers. Nov Appro in Can Study. 6(2). NACS.000631. 2021. DOI: 10.31031/NACS.2021.06.000631

Copyright@ Daniel Borsch, This article is distributed under the terms of the Creative Commons Attribution 4.0 International License, which permits unrestricted use and redistribution provided that the original author and source are credited.

\section{A Review of the Potential Preventive Effects of Adiponectin in Major Cancers}

Kevin Pak, Johnny Dang, Michaela LeDonne, Emily Converse, Niki Nourmohammadi, Pranav Cannanbilla, Dario Fiorelli, Connor Foote, Tanner Foster, Aaron Grossberg, Kelsie Hendrick, Camilla Herbin, Marcel Mio, Minh Nguyen, Alexander Pappas, James Pirraglia, Allison Quirk, John Sinclair, Amitabha Ray and Daniel Borsch*

Lake Erie College of Osteopathic Medicine at Seton Hill, USA

\begin{abstract}
Adiponectin is a hormone-like cytokine (or adipokine) predominantly secreted from fat cells. Adiponectin has anti-inflammatory properties and inversely correlates with body fat mass, including visceral adiposity. This cytokine is present in different isoforms and may mediate its biological functions mainly through AdipoR1 and AdipoR2 receptors. It is thought that adiponectin has been associated with the pathological processes of diabetes, heart disease, and cancer. In our review of PubMed literature, we found interesting data regarding the effect of adiponectin levels on many types of cancer. Several studies have observed a protective role of adiponectin in some types of cancer. In endometrial, colon, kidney, postmenopausal breast cancer, and leukemia, there appears to be an inverse relationship between adiponectin levels and cancer. Understanding adiponectin's biological connection with other hormones (e.g., insulin, insulin-like growth factors, leptin), as well as its modulation in pre-neoplastic states and malignancy to exploit its beneficial effects, could reveal potential new strategies for the management of certain types of cancer.
\end{abstract}

\section{Introduction}

Adiponectin is a hormone-like cytokine (adipokine) mainly released from fat cells. It is believed that adiponectin has anti-inflammatory properties and inversely correlates with body's adipose tissue, particularly visceral adiposity. This cytokine is present in at least three different isoforms: Low Molecular Weight (LMW) trimers, Medium Molecular Weight (MMW) hexamers, and High Molecular Weight (HMW) oligomers. It is suggested that these isoforms mediate their biological effects mainly through two receptors: AdipoR1 and AdipoR2 (Figure $1 \&$ 2) [1]. Lower levels of adiponectin have been reported to be associated with insulin resistance/diabetes, cardiometabolic disorders, and certain cancers. This short review, modified from a poster, provides a focused analysis of the biological role of adiponectin in major cancers. The poster web-link contains a detailed account of the references [2].

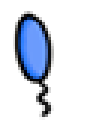

Monomer

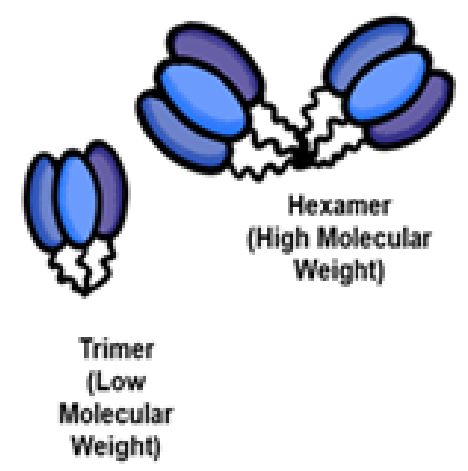

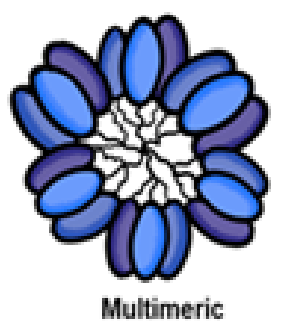

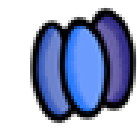

Globular
Figure 1: Structural organizations of different adiponectin isoforms. 


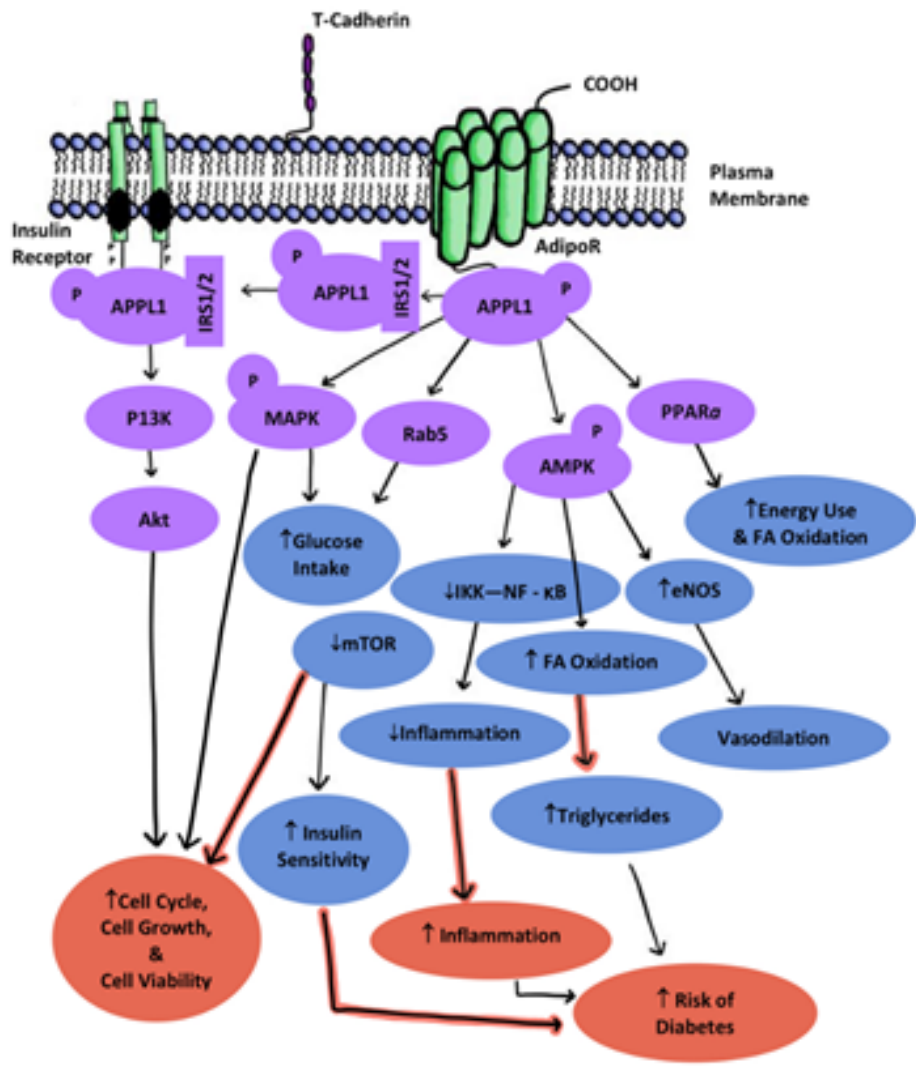

Figure 2: Adiponectin-linked intracellular signaling molecules and their association with different pathophysiological conditions.

AdipoR: Adiponectin Receptors; Akt: A Serine/Threonine Kinase or Protein Kinase B; AMPK: Adenosine Monophosphate-Activated Protein Kinase; APPL1: Adaptor Protein; Adiponectin Receptor Binding Protein; eNOS: Endothelial Nitric Oxide Synthase; FA: Fatty Acid; IKK: Inhibitor of Nuclear Factor-kB Kinase Complex; NF-kB: Nuclear Factor Kappa-Light-Chain-Enhancer of Activated B Cells; IRS1/2: Insulin Receptor Substrate 1 and 2; MAPK: Mitogen-Activated Protein Kinase; mTOR: Mechanistic Target of Rapamycin; P: Phosphorylation; PPARa: Peroxisome Proliferator-Activated Receptor Alpha; P13K: Phosphoinositide 3-kinases, also called phosphatidylinositol 3-kinases; Rab5: a small GTPase (GTP: guanosine-5'-triphosphate); T-cadherin: it may act as a receptor for adiponectin.

\section{Methods and Methods}

To better understand the role of adiponectin in various cancers, a review of PubMed literature was performed. In the early part of 2020, a targeted search with keywords "adiponectin" + respective cancer of interest yielded a total of 1322 human, animal, and cellline research study articles. For human studies, only clinical and epidemiological papers examining aggressiveness of primary tumors in relation to serum levels of adiponectin were chosen for review. Furthermore, a similar scheme was followed for invivo/animal studies. For in-vitro/cell-line studies, only articles examining the effect of adiponectin on tumor cell proliferation or intracellular signaling were selected for review. All review articles were filtered from this survey. In total, 191 articles met the criteria for this review [2]; (Table 1).

Table 1: Review of adiponectin and its anti-neoplastic effects in different cancers.

\begin{tabular}{|c|c|c|c|}
\hline Cancer site & Human Studies & Animal Studies & Cell-line Studies \\
\hline Breast & $15|3| 6$ & $7|1| 0$ & $0|4| 0$ \\
\hline Endometrium & $15|0| 2$ & $0|0| 0$ & $6|0| 0$ \\
\hline Ovary & $2|0| 1$ & $1|0| 1$ & $1|0| 0$ \\
\hline Prostate & $9|4| 6$ & - & $9|0| 0$ \\
\hline Thyroid & $2|1| 2$ & $3|0| 0$ & $5|1| 0$ \\
\hline Colorectal & $3|1| 1$ & $3|0| 0$ & $3|0| 0$ \\
\hline Liver & $9|3| 0$ & $1|2| 1$ & $3|0| 0$ \\
\hline Pancreas & $4|0| 0^{*}$ & $0|3| 1^{* *}$ & \\
\hline
\end{tabular}




\begin{tabular}{|c|c|c|c|}
\hline Renal & $12|1| 0$ & - & $1|0| 0$ \\
\hline Lung & $6|0| 2$ & $0|0| 0$ & $4|0| 0$ \\
\hline Skin & $0|0| 1$ & $1|0| 0$ & $2|0| 0$ \\
\hline Leukemia & $9|0| 0$ & - & - \\
\hline Lymphoma & $0|5| 0$ & - & - \\
\hline
\end{tabular}

Blue: Inverse relationship between adiponectin and cancer

Red: Direct correlation between adiponectin and cancer

Green: No correlation

* Pre-diagnosis cases

** Post-diagnosis cases

\section{Breast Cancer}

Fourteen studies examining adiponectin levels and their correlation with breast cancer risk found that serum adiponectin was inversely associated to the risk. Serum adiponectin levels were also inversely related to tumor adiponectin levels in breast cancer patients. On the other hand, three studies found that adiponectin levels increased with risk factors associated with breast cancer. Furthermore, six studies found that there was no significant difference between adiponectin levels in breast cancer patients and control groups.

Animal studies reviewed also demonstrated adiponectin's protective role in breast cancer. Of the eight animal studies that examined the correlation between adiponectin levels and cancer, seven studies found that low adiponectin levels were correlated to increased tumor growth, while one showed the opposite effect. Additionally, an analysis of cell culture studies showed that, at higher levels of adiponectin, genes that are considered proapoptotic were stimulated, and anti-apoptotic genes were inhibited. Specifically, apoptotic rates were increased in MDA-MB-231, MCF-7 and SK-BR-3 cell-lines. Adiponectin can also inhibit endothelial cell proliferation by $15 \%$ and migration by $82 \%$. Globular adiponectin has been shown to have protective effects in breast cancer by decreasing the cell number of MCF-7 cells with an increase in the number of cells in G0/G1 phase, decreasing the number of cells in S phase and increasing caspase- 3 activity.

\section{Ovarian and Endometrial Cancer}

For human, animal, and cell-line studies examining the correlation between endometrial cancer and adiponectin levels, the majority revealed decreased adiponectin levels in the presence of endometrial cancer. Among the human, animal, and cell studies investigating the relationship between adiponectin levels and ovarian cancer, a consistent trend was not identified. For both ovarian and endometrial cancers, there was a limited number of studies performed investigating the neoplastic relationship with adiponectin levels.

\section{Prostate Cancer}

The data show that low levels of adiponectin were associated with higher Gleason scores. Of note, the Gleason score is a grading system utilized to assess the extent to which biopsy cells look like normal prostate tissue under the microscope. Higher scores represent higher malignant growth patterns and metastatic risk. Few studies reported that adiponectin levels were not a reliable diagnostic or screening tool for prostate cancer. It was also suggested that peri-prostatic white adipose tissue inflammation may be connected with lower adiponectin levels and more aggressive cancer.

\section{Thyroid Cancer}

Among human studies, elevated adiponectin levels were found to be negatively associated with differentiated thyroid cancer compared to benign thyroid disease. However, other results indicated that adiponectin had no significant association in patients with papillary thyroid cancer and medullary thyroid cancer. Among cell-line studies, over-expression of adiponectin receptors was observed in papillary thyroid cancer.

\section{Colon Cancer}

For colon cancer, three human studies found higher levels of adiponectin to be protective against cancer, whereas one publication showed high levels of adiponectin with cancer promotion, although no association was also suggested by one study. On the other hand, animal studies revealed that high levels of adiponectin were protective against cancer. Overall, this observation supported data from five reported cell-line studies. Nevertheless, one cell-line study suggested that adiponectin promoted cancer.

\section{Liver Cancer}

The role of adiponectin in human hepatic cancers appears to be complex. Indeed, some studies have suggested that higher circulating levels of adiponectin were protective against Hepatocellular Carcinoma (HCC) in patients with liver cirrhosis. Lower levels of adiponectin have also been shown to be associated with worse histological grades of HCC. Other studies, however, suggested that high levels of adiponectin were associated with an increased risk of developing HCC or worse outcomes in HCC patients.

Few in-vivo studies have linked low levels of adiponectin with the development of HCC. Adiponectin has also been found to prevent tumor metastasis in a mouse model by suppressing angiogenesis. Additionally, an in-vitro study suggested that adiponectin inhibited leptin-mediated proliferation of HCC. Moreover, adiponectin has also been shown to be protective by promoting apoptosis. 


\section{Pancreatic Cancer}

The four human trials that investigated pre-diagnostic adiponectin levels found an association between high adiponectin levels and a decreased risk of pancreatic cancer. In contrast, three research studies that examined post-diagnostic adiponectin levels showed adiponectin levels were elevated in pancreatic tumor cases compared with control groups. Overall, animal studies yielded contradicting results: two demonstrated that adiponectin decreased pancreatic tumor size, while another study suggested adiponectin level was correlated with an increase in pancreatic tumor size. One study found that adiponectin contributed to pancreatic tumor growth specifically by inhibiting apoptosis. Among limited cell culture studies, one group of investigators observed that adiponectin was capable of inducing apoptosis, while the receptors for adiponectin were minimally expressed in pancreatic cancer cell-lines.

\section{Renal Cancer}

In general, decreased serum adiponectin levels were associated with an increased risk of renal cell carcinoma and/or an increased risk of metastasis. Nevertheless, one paper found a direct and significant correlation of adiponectin with renal cell carcinoma in African American people and females, and a non-significant correlation in Caucasian males.

\section{Lung Cancer}

Current evidence fails to reveal any prognostic or diagnostic associations between serum adiponectin levels and lung carcinoma. However, studies showed that adiponectin had an inhibitory effect on Non-Small Cell Lung Cancer (NSCLC) cell proliferation in a time- and dose-dependent manner via apoptosis or cell cycle arrest through modulation of AdipoR1 and AdipoR2.

\section{Skin Cancer}

Adiponectin was shown to be downregulated in keratoacanthoma, a variant of squamous cell carcinoma. Moreover, adiponectin concentration was also found to be negatively correlated with insulin resistant induced melanoma. In another study, high fat diets were shown to decrease plasma/serum levels of adiponectin and increase inflammation in UV-irradiated mouse model, resulting in a greater chance of the mice contracting skin cancer. A single human study noted an inverse association of serum adiponectin with melanoma, although the significance was unclear.

\section{Leukemia and Lymphoma}

Epidemiological studies demonstrated that adiponectin levels exhibited an inverse correlation with an incidence among patients with leukemia. Furthermore, our research of the literature also suggested a positive correlation of serum adiponectin levels in patients diagnosed with lymphoma. No in-vivo/animal or in-vitro/ cell-line study results regarding leukemia or lymphoma matched our survey criteria.

\section{Discussion and Conclusion}

Our review of PubMed articles based on the search criteria of 'adiponectin' and 'cancer' was inconclusive. While in-vivo/ animal studies and in-vitro studies generally observe an inverse relationship between cancer aggressiveness and adiponectin levels, suggesting a protective role, the connection between adiponectin and cancer in humans appears to be much more complex (Figure 3).

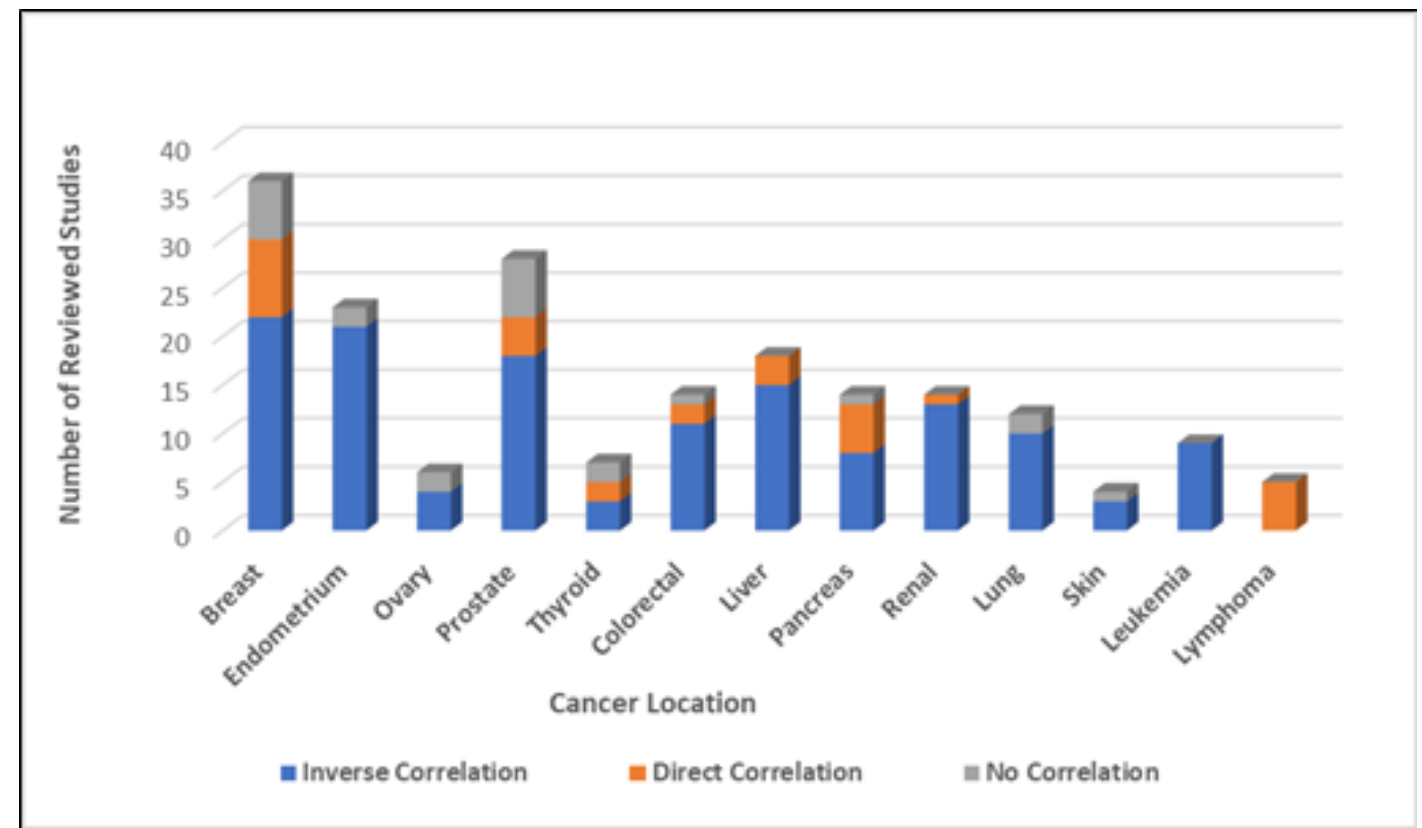

Figure 3: Summary of adiponectin and its anti-neoplastic effects.

In this bar graph, the blue indicates the number of articles reporting an inverse relationship between adiponectin levels and the organ related cancer. The orange indicates a direct/positive relationship between adiponectin levels and the organ related 
cancer; and the grey denotes the number of articles that did not observe any correlations. For instance, in liver cancer, a direct relationship was noticed with higher levels of adiponectin being associated with a more aggressive cancer, while no association could be demonstrated in many other cancers. In some cancers, however, such as endometrial, colon, kidney, postmenopausal breast cancer, and leukemia, there appears to be a protective effect exerted by adiponectin against pathological processes, suggesting a role for adiponectin in the management of and treatment strategy for certain types of cancer. To exploit the promising beneficial effects of adiponectin, it will be crucial to better understand both its biological connection with other hormones (e.g., insulin, insulin- like growth factors, leptin), as well as the modulation of adiponectin and adiponectin receptor levels in premalignant and malignant states.

\section{References}

1. Nigro E, Daniele A, Salzillo A, Ragone A, Naviglio S, et al. (2021) AdipoRon and other adiponectin receptor agonists as potential candidates in cancer treatments. Int J Mol Sci 22(11): 5569.

2. Nourmohammadi N, Converse E, LeDonne M, Cannanbilla P, Dang J, et al. (2020) Adipose tissue-released hormone adiponectin: A review of its anti-neoplastic effects. Lake Erie College of Osteopathic Medicine-Seton Hill, Greensburg, PA, USA, [Poster, POMA Conference]. 